\title{
Medical Image of the Week: Massive Abdominal Aortic Aneurysm
}

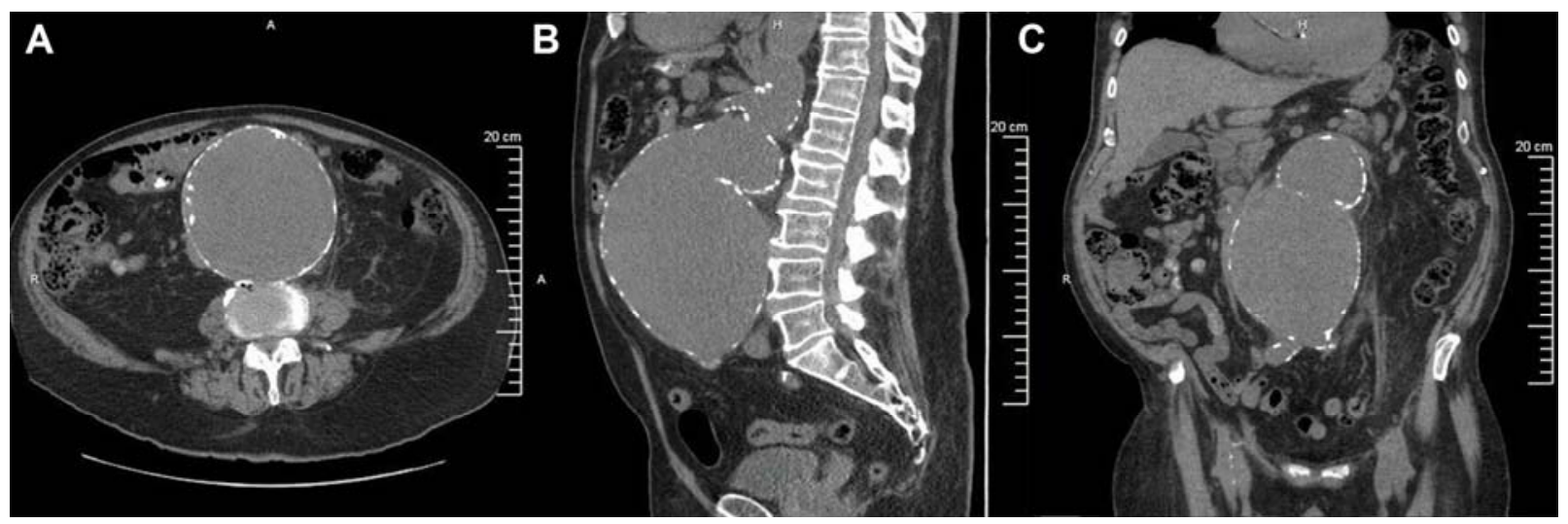

Figure 1. Non-contrast CT A) axial, B) sagittal, and C) coronal views demonstrate a massive abdominal aortic aneurysm measuring $12.5 \mathrm{~cm}$ wide at maximal diameter.

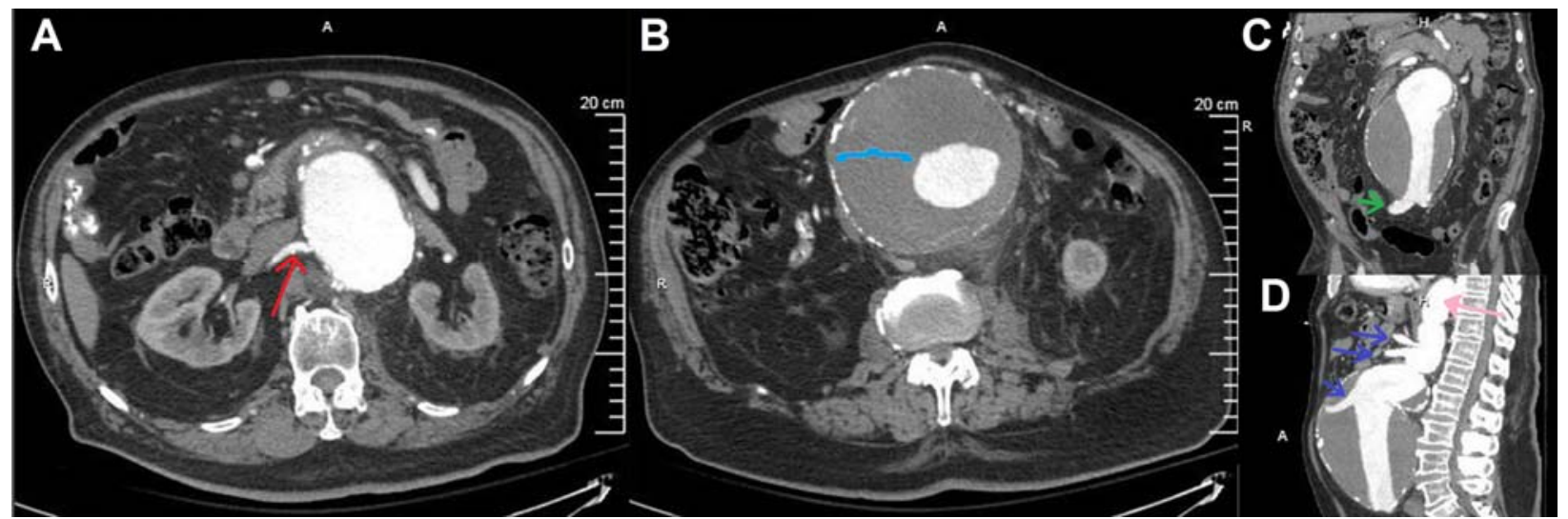

Figure 2. Representative images from a CT-angiogram shows A) upper and B) lower abdominal axial sections showing renal artery involvement (red arrow) and substantial intramural thrombus (light blue brace). C) Coronal view demonstrates fusiform dilation of the iliacs (green arrow) and D) sagittal view demonstrates involvement of the thoracoabdominal aorta (pink arrow) and all major arterial branches of the abdominal aorta (celiac trunk, superior and inferior mesenteric arteries; dark blue arrows).

An 88 year-old presented to the emergency department with left flank and lower back pain as well as lower abdominal fullness. The fullness had started 2 days prior, but the left flank pain acutely started in the early morning before presenting. He had a history of unmedicated hypertension, hyperlipidemia, and mild vertigo. His review of systems was positive for chills and difficulty urinating but no hematuria. He was a non-smoker, and had undergone orthopedic surgeries but had otherwise avoided emergent hospitalizations. 
On exam, vitals were unremarkable; there was no flank nor costovertebral angle tenderness; however, a midline pulsatile mass was present. An initial non-contrast CT abdomen/pelvis revealed a massive abdominal aortic aneurysm (AAA, Figure 1). Follow-up CT angiogram of the AAA can be seen in Figure 2. Upon further questioning, he had undergone a research study some 30 years earlier involving ultrasound to screen for AAA and was told he did not have one at the time.

AAA's occur in 4-9\% of the population (1-3) because of the diminished elastin in the infrarenal aorta. Inciting or etiologic factors include inflammatory, genetic and biochemical mediators, with positive risk factors including white race, atherosclerosis, smoking, male gender, hypertension, personal history of other arterial aneurysms, family history of AAA's, and advancing age. Screening all men aged 65-79 has been shown to reduce mortality (2) despite the non-trivial mortality associated with elective AAA repair (3). Only $1 \%$ of 65 year-old men with a negative ultrasound will go on to develop an AAA (2).

The feared and fatal complication of AAA is rupture, and occurs in 10,500 $\pm 1,500$ patients yearly in the U.S.A., with larger AAA's posing higher annual risk of rupture (1$3)$. Emergent surgical repair mortality in the $30-50 \%$ that survive a rupture long enough to go to the operating room is roughly $50 \%$.

The extensive nature of this patient's aneurysm would have made for a nearlyimpossible surgery, with operative mortality estimates between $15 \%$ using the British Aneurysm Repair Score (3) to $50 \%$ based on clinical opinion. This dissuaded the patient, his family, and vascular surgery team from pursuing elective repair. The patient desired discharge with pain medications and stricter blood pressure control with outpatient follow-up.

Michael Larson, M.D., Ph.D.

Tucson Hospitals Medical Education Program

Tucson, AZ, USA

\section{References}

1. Lederle FA. Ultrasonographic screening for abdominal aortic aneurysms. Ann Intern Med. 2003 Sep 16;139(6):516-22. [CrossRefl [PubMed]

2. Cosford PA, Leng GC. Screening for abdominal aortic aneurysm. Cochrane Database Syst Rev. 2007 Apr 18;(2):CD002945. [CrossRef] [PubMed]

3. Grant SW, Hickey GL, Grayson AD, Mitchell DC, McCollum CN. National risk prediction model for elective abdominal aortic aneurysm repair. Br J Surg. 2013 Apr;100(5):645-53. [CrossRefl [PubMed] 